\title{
Structure of human Fe-S assembly sub-complex
}

Seth A. Cory ${ }^{\mathrm{a}}$, Jonathan G. Van Vranken ${ }^{\mathrm{b}}$, Edward J. Brignole ${ }^{\mathrm{c}, \mathrm{d}}$, Shachin Patra ${ }^{\mathrm{a}}$, Dennis R. Winge $^{\mathrm{b}, \mathrm{e}}$, Catherine L. Drennan ${ }^{\mathrm{c}, \mathrm{d}, \mathrm{f}}$, Jared Rutter ${ }^{\mathrm{b}, \mathrm{g}}$ and David P. Barondeau ${ }^{\mathrm{a}}$

${ }^{a}$ Department of Chemistry, Texas A\&M University

${ }^{b}$ Department of Biochemistry, University of Utah School of Medicine

${ }^{\mathrm{c} D e p a r t m e n t}$ of Chemistry, Massachusetts Institute of Technology

${ }^{\mathrm{d}}$ Howard Hughes Medical Institute, Massachusetts Institute of Technology

${ }^{\mathrm{e}}$ Department of Medicine, University of Utah School of Medicine

fDepartment of Biology, Massachusetts Institute of Technology

${ }^{\text {g} H o w a r d ~ H u g h e s ~ M e d i c a l ~ I n s t i t u t e, ~ U n i v e r s i t y ~ o f ~ U t a h ~ S c h o o l ~ o f ~ M e d i c i n e ~}$

Iron-sulfur (Fe-S) clusters are essential cofactors for all organisms. In eukaryotes, Fe-S cluster are primarily synthesized in the mitochondria and distributed to target proteins involved in substrate activation, oxidative respiration, and gene regulation. ${ }^{1}$ At the center of the Fe-S cluster biosynthetic complex, the cysteine desulfurase (NFS1) and LYR protein (ISD11) form a tight complex and catalyze the conversion of cysteine to alanine in which persulfide sulfur is delivered via a mobile loop to the scaffold protein, ISCU2. ${ }^{1}$ Additionally, FXN appears to act as an allosteric activator of this process. ${ }^{2,3}$ On ISCU2, persulfide sulfur, $\mathrm{Fe}^{2+}$, and electrons are assembled to form [2Fe-2S]. Each of the aforementioned proteins are involved in human disease. ${ }^{1}$ Additionally, recent functional and proteomic studies have revealed acyl-carrier protein (ACP) as an essential component of Fe-S cluster biosynthesis and as an interacting partner of ISD11 and other LYR proteins respectively. ${ }^{4,5}$ Interestingly, ACP and ISD11 are eukaryotic specific adaptors for the Fe$\mathrm{S}$ cluster biosynthetic complex, and to date, the structural basis for their essentiality has yet to be described. We have determined a $3.09 \AA$ and $15 \AA$ x-ray crystal and electron microscopy structures respectively of human NFS1-ISD11 in complex with E. coli ACP. Our structure reveals a dramatically different global architecture in comparison to all previously determined cysteine desulfurase structures. To support this architecture, we also interrogated protein-protein interfaces in vivo using S. cerevisiae. Overall, the structure reveals that ACP threads its lipid bound cofactor into ISD11 providing stabilization and a structural framework the assembly of a new cysteine desulfurase global architecture. We hypothesize that this new architecture facilitates the use of function-control elements such as FXN, and that ACP interacts with all LYR proteins via a lipid insertion motif serving as a regulatory element of both oxidative respiration and $\mathrm{Fe}-\mathrm{S}$ cluster biosynthesis.

(1) Lill, R.; Mühlenhoff, U. Annual Review of Biochemistry 2008, 77, 669.

(2) Tsai, C.-L.; Barondeau, D. P. Biochemistry 2010, 49, 9132.

(3) Bridwell-Rabb, J., et al. Biochemistry 2014, 53, 4904.

(4) Huttlin, E. L., et al. Cell 2015, 162, 425.

(5) Van Vranken, J. G., et al. Elife 2016, 5. 